\title{
Halt and recovery of illusory motion perception from peripherally viewed static images
}

\author{
Erika Tomimatsu • Hiroyuki Ito $\cdot$ Shoji Sunaga • \\ Gerard B. Remijn
}

Published online: 4 May 2011

(C) Psychonomic Society, Inc. 2011

\begin{abstract}
We quantitatively investigated the halt and recovery of illusory motion perception in static images. With steady fixation, participants viewed images causing four different motion illusions. The results showed that the time courses of the Fraser-Wilcox illusion and the modified Fraser-Wilcox illusion (i.e., "Rotating Snakes") were very similar, while the Ouchi and Enigma illusions showed quite a different trend. When participants viewed images causing the Fraser-Wilcox illusion and the modified Fraser-Wilcox illusion, they typically experienced disappearance of the illusory motion within several seconds. After a variable interstimulus interval (ISI), the images were presented again in the same retinal position. The magnitude of the illusory motion from the second image presentation increased as the ISI became longer. This suggests that the same adaptation process either directly causes or attenuates both the Fraser-Wilcox illusion and the modified FraserWilcox illusion.
\end{abstract}

Keywords Fraser-Wilcox illusion - Adaptation · Rotating snakes $\cdot$ Ouchi illusion $\cdot$ Enigma illusion

Some static images are known to cause illusory motion perception. Fraser and Wilcox (1979) found that a static image consisting of sectors painted in gradations from black to white generates strong illusory motion (Fig. 1a). This type of stimulus tends to produce illusory rotation in a direction from dark to light (the Fraser-Wilcox [FW] illusion: Faubert \& Herbert, 1999; Naor-Raz \& Sekuler, 2000), a motion direction that is determined by genetic

E. Tomimatsu $(\bowtie) \cdot H$. Ito $\cdot S$. Sunaga $\cdot$ G. B. Remijn

Faculty of Design, Kyushu University,

4-9-1, Shiobaru,

Minami-ku, Fukuoka-shi 815-8540, Japan

e-mail: tomimatsu@kyudai.jp factors according to Fraser and Wilcox. The strength of the illusion depends on the stimulus contrast and the retinal eccentricity on which the stimulus image is presented (Naor-Raz \& Sekuler, 2000).

In a later study, Kitaoka and Ashida (2003) proposed a type of stimulus related to that described by Fraser and Wilcox (1979), but composed of a different luminance profile. Although Kitaoka and Ashida (2007) called it the "optimized" Fraser-Wilcox illusion, we will call it the "modified" FW illusion, because it is not clear whether its origin is the same as that of the FW illusion. Kitaoka and Ashida (2003) reported that strong illusory motion was acquired through a stimulus configuration consisting of four segmented luminance steps: black, dark gray, white, and light gray (Fig. 1b). They suggested that the direction of the perceived motion is from black, to dark gray, white, and light gray - a luminance profile that, for example, produced the "Rotating Snakes" (Kitaoka, 2003) illusion. Studies have quantitatively confirmed that the motion in this illusion appears more in peripheral vision (Beer, Heckel, \& Greenlee, 2008; Hisakata \& Murakami, 2008) and that the illusion is weaker for senior observers (Billino, Hamburger, \& Gegenfurtner, 2009). One characteristic common to both the FW illusion and the modified FW illusion is that steady fixation weakens the illusory motion, and eventually halts it within several seconds. In this article, we focused on the time course of the changes in the strength of illusory motion.

Several studies have proposed hypotheses on the mechanisms inducing illusory motion from static images. Conway, Kitaoka, Yazadanbakhsh, Pack, and Livingstone (2005) suggested that the modified FW illusion is caused by differences in response timing of cells due to different luminance contrasts. They physiologically demonstrated that V1 and MT cells show shorter-latency responses to 


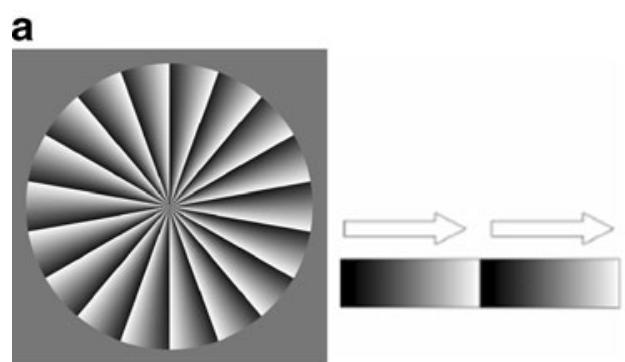

Fig. 1 Two types of luminance profiles that effectively cause illusory motion. a. A stimulus composed of sectors that gradually change from dark to light, used by Fraser and Wilcox (1979). The illusory motion direction tends to go from dark to light (Faubert \& Herbert, 1999;

higher-contrast elements (white and black) than to lowercontrast elements (light gray and dark gray). Accordingly, direction-selective neurons in macaque visual cortex produced the expected directional responses to high- and low-contrast element pairs (from white to light-gray and from black to dark-gray elements). Conway et al. also showed physiological evidence indicating that white/dark-gray and black/light-gray pairs cause "reversed phi" (Anstis, 1970), resulting in motion perception from dark gray to white and from light gray to black elements. However, how motion detectors continue to signal motion for several seconds from the initial timing difference is not clear.

Murakami, Kitaoka, and Ashida (2006) hypothesized that the modified FW illusion is caused by involuntary small eye movements. They found that there was a positive correlation between fixation instability and the magnitude of the illusory motion seen in "Rotating Snakes." However, they did not find any correlation between the probabilities of microsaccades and the illusion's strength. They suggested that an asymmetry in the cell responses to changing contrasts, caused by retinal image shifts from drift eye movements, generates stronger motion signals in a particular direction. Beer et al. (2008) also suggested the involvement of fixation instability with the illusory motion. Although they also did not find any effect of microsaccades, they suggested that the magnitude of drift eye movements correlated with illusory motion ratings. They measured fixation instability in a time course for $4 \mathrm{~s}$ after the onset of the stimulus. The data showed that the fixation instability for the first second was greater than that for the subsequent $2 \mathrm{~s}$. However, judging from their Fig. 3, the amount of decrease in their measure of drift was only about $10 \%$, and the amount of drift even increased during the fourth second. The eye movement theory alone thus does not explain how illusory motion from static images gradually stops in several seconds.

Backus and Oruç (2005) proposed another hypothesis for the mechanism, based on local adaptation to luminance and contrast. During these two adaptation processes, the summed responses to the images in the visual system are b

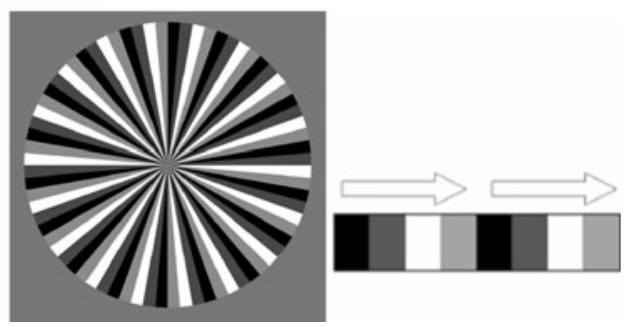

Naor-Raz \& Sekuler, 2000). b. A stimulus composed of four segmented luminance steps, proposed by Kitaoka and Ashida (2003). The illusory motion direction tends to go from black to dark gray, and from white to light gray

considered to cause a phase shift of the fundamental spatial frequency. They noted that the modified FW illusion was mainly due to rapid contrast adaptation and that the FW illusion was mainly due to slow luminance adaptation. The adaptation hypothesis explains how the illusory motions tend to stop with steady fixation and restart after a saccade or by moving the image (Backus \& Oruç, 2005; Faubert \& Herbert, 1999). Interestingly, Backus and Oruç noted that the illusory motion stops after about 6-8 $\mathrm{s}$ of steady fixation and that when refixating a previously fixated point, the time required for motion to stop increases with the time away from the point. This observation may include direct evidence for an effect of retinotopic adaptation on illusory motion, but it is only qualitatively described.

The first purposes of the present study were to provide a detailed description of the whole time course of the strength of illusory motion and to quantitatively examine the observation of Backus and Oruç (2005) with regard to the stimuli described above. We studied both the FW illusion and the modified FW illusion. Our measurements included the start, (perceived) halt, pause, restart, and (perceived) rehalt phases of the two types of illusory motion. Following Backus and Oruç's reasoning, we hypothesized that the gradual decrease in perceived illusory motion during a fixation reflects the progress of local luminance and contrast adaptation. The halt of the illusory motion may eventually be caused by saturation. Accordingly, the recovery time of the illusory motion in relation to the amount of time spent looking away from the illusion may indicate gradual recovery from saturation. Collecting quantitative evidence will be critical to verifying this proposed adaptation process in illusory motion from static images. To our knowledge, however, no report has provided complete descriptions of the continuous changes in the illusion strength over $20 \mathrm{~s}$ (36 s under the longest condition here). Especially, recovery from the halt of illusory motion has never been psychophysically investigated.

Our second purpose was to compare the time courses of the illusions. With the comparison, we examined whether the effect of adaptation on the illusion strength is common 
between the two types of illusions. Thus, we compared the whole time courses noted above. We also compared the time courses with those of other motion illusions - that is, the Ouchi illusion (also known as the Spillmann illusion; Spillmann, Heitger, \& Schuller, 1986) and the Enigma illusion (Leviant, 1996). The Ouchi illusion is an image consisting of a disk that is filled with black and white checks with an orthogonally oriented checkerboard in surround. It produces the perception of drift or smooth motion. The Ouchi illusion arises when the eye or the figure smoothly moves (Fermüller, Pless, \& Aloimonos, 2000). The figure causing the Enigma illusion consists of spokes and concentric rings in which illusory "streaming" can be observed. The streaming effect of the Enigma illusion is said to be related to microsaccades during fixation (Troncoso, Macknik, Otero-Millan, \& MartinezConde, 2008), although Hamburger (2007) showed that the Enigma illusion was not produced but instead enhanced by microsaccades. Both the Ouchi and Enigma figures include black and white stripes. The effect of adaptation to local luminance and contrast may therefore also appear in these illusions, making it worth while to include them in additional comparisons with the FW illusion and the modified FW illusion.

In short, this article aims to quantitatively test the possible effect of local luminance and contrast adaptation on changes in illusory motion from static images and to examine whether the effect is common for the motion illusions reported by Fraser and Wilcox (1979) and Kitaoka and Ashida (2003), in comparison with the same effect on Ouchi and Enigma illusions.

\section{Method}

Participants

Eleven participants took part in the experiment. One of them was the first author, and the others were graduate or undergraduate students (age 21-25 years) who were naïve as to the purpose of this study. All of the participants had normal or corrected-to-normal vision. Five of the participants, along with the first author, observed both the FW illusion and the modified FW illusion. The other 5 participants, again with the first author, observed both the Ouchi and Enigma illusions.

\section{Apparatus}

The stimuli were presented on a CRT monitor (Mitsubishi RDF223G) controlled by a computer (Compaq Evo) and graphic equipment (AV Tachistoscope, Iwatsu, IS-703). The participants observed the stimuli from a distance of $60 \mathrm{~cm}$ in a darkened room. Their heads were stabilized with a chinrest.

\section{Stimuli}

We created two types of illusory motion stimuli, from here on called the $F W$ stimulus and the modified-FW stimulus (Fig. 2a and b). They had two different luminance profiles, corresponding to the stimuli proposed by Fraser and Wilcox (1979) and by Kitaoka and Ashida (2003), respectively. The FW stimulus was a considerably simplified version of the stimulus used by Fraser and Wilcox. Their luminance gradation was replaced by four segmented luminance steps of $0,58.3,117.7$, and $175 \mathrm{~cd} / \mathrm{m}^{2}$, respectively, with a mean luminance of $87.5 \mathrm{~cd} / \mathrm{m}^{2}$. The modified-FW stimulus was produced by replacing the order of the segments in the FW stimulus as shown in Fig. 2. Thus, although the annuli in both stimuli included sectors in the same four luminance steps, only the spatial order differed between the two. The luminance profile was repeated nine times in the annuli. The stimulus annulus $\left(4.6^{\circ}\right.$ or $6.1^{\circ}$ in inner or outer diameter) and a fixation point were presented at the center of a mid-gray background $\left(20.7^{\circ} \times 20.7^{\circ}\right)$. The luminance of the background was the same as the mean luminance of the stimulus. The distance between the fixation point and the stimulus center was $14.3^{\circ}$.

We also used two stimuli consisting of an Ouchi figure and an Enigma ring. The Ouchi figure consisted of a horizontally oriented checkerboard superimposed with a vertically oriented checkered disk. The disk or checkerboard was composed of repeated black and white small squares $\left(1.8^{\circ} \times 0.6^{\circ}\right.$ or $0.6^{\circ} \times$ $1.8^{\circ}$ for each square), with luminances of 0 and $175 \mathrm{~cd} / \mathrm{m}^{2}$, respectively. The size of the checkerboard subtended $20.7^{\circ} \times$ $20.7^{\circ}$. The disk was $9.6^{\circ}$ in diameter. A red fixation cross was placed at the center of the image. The Enigma figure a
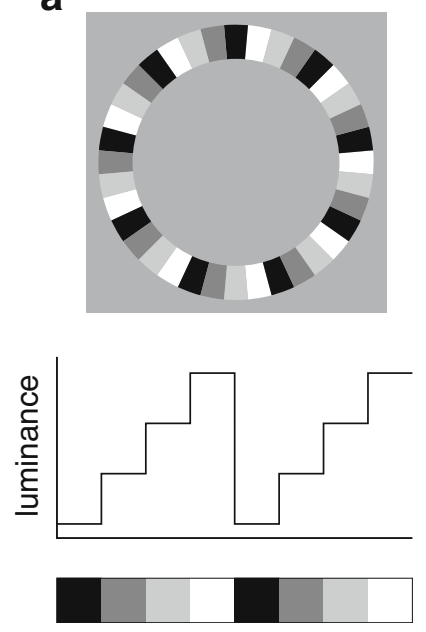

b
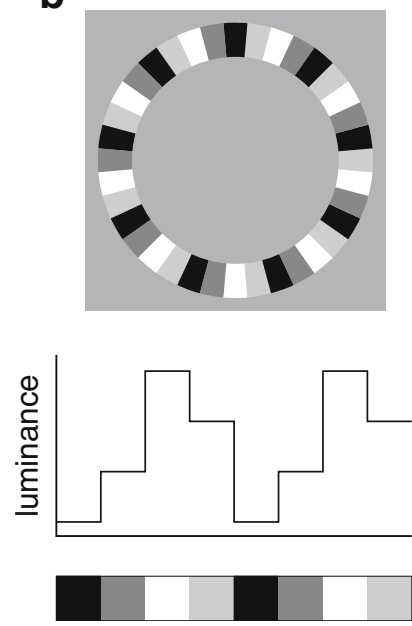

Fig. 2 Two types of stimuli used in the experiment. a. The FW stimulus, simplified from the stimulus used by Fraser and Wilcox (1979). b. The modified-FW stimulus, simplified from the stimulus that Kitaoka and Ashida (2003) proposed. Both stimuli have four segmented luminance steps 
consisted of black and white spokes $\left(0\right.$ and $\left.175 \mathrm{~cd} / \mathrm{m}^{2}\right)$ with a centered disk $\left(117.7 \mathrm{~cd} / \mathrm{m}^{2}\right)$ and three concentric rings $\left(58.3 \mathrm{~cd} / \mathrm{m}^{2}\right.$ for the outer and middle rings, and $87.5 \mathrm{~cd} / \mathrm{m}^{2}$ for the inner ring). The inner diameter of the middle ring was $9.6^{\circ}$. Here too, a red fixation cross was superimposed at the center of the image.

\section{Procedure}

A sample trial sequence under the FW and modified-FW stimulus conditions is shown in Fig. 3. Following the presentation of a fixation point for $5 \mathrm{~s}$, one of the stimuli was presented for $10 \mathrm{~s}$ (the first image presentation), and then erased. After one of eleven ISIs $(10,30,50,100,200$, $400,800,1,000,4,000,9,000$, and $16,000 \mathrm{~ms}$ ), the same image was presented again (the second image presentation) at the same retinal position as in the first image presentation. After $10 \mathrm{~s}$, a display of random dots followed the second image presentation in order to weaken the perception of an afterimage. In a control condition, it was immediately presented again at a different location after erasing the first image.

The stimulus was randomly presented on the left, on the right, or just below the fixation cross in each trial. The two types of stimuli and the 11 ISIs were mixed in a block, and their orders were also randomized. To investigate recovery from the motion's halt following inserted ISIs, participants pressed one of two response keys when they saw motion in the presented stimulus, according to whether they saw clockwise or counterclockwise rotation. They pressed a third key when the illusory motion stopped. The time interval between pressing the "clockwise" or "counterclockwise" key and pressing the "stopped" key was calculated as the duration of

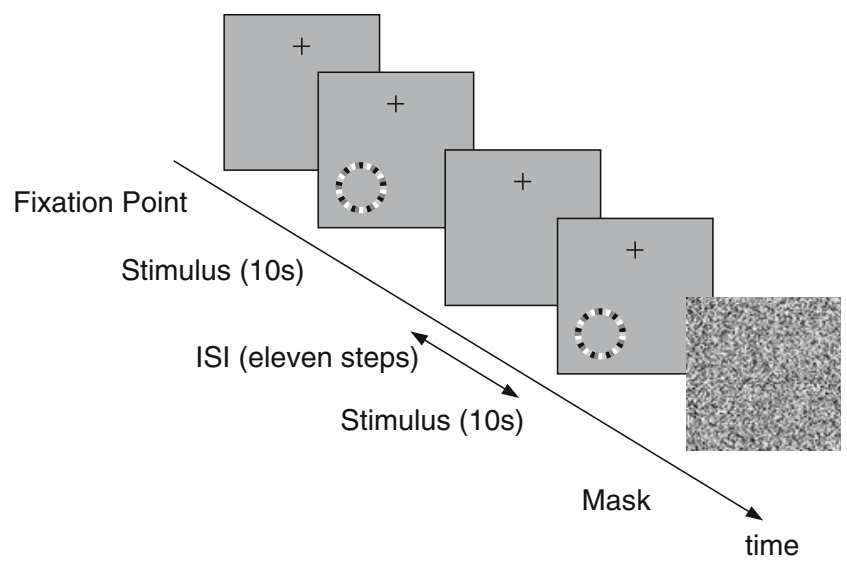

Fig. 3 Schematic illustration of the experimental paradigm. First, a fixation cross was presented on a display with a mid-gray background for $5 \mathrm{~s}$. Then one of the two stimulus types (FW or modified FW) was presented for $10 \mathrm{~s}$. After a blank interval (11 steps of interstimulus intervals: $0.01-16 \mathrm{~s}$ ), the stimulus was again presented for $10 \mathrm{~s}$. When illusory motion started the participants pressed a button, and they pressed another when the illusory motion stopped perceived illusory motion. Participants could press the keys at any time, whenever they saw illusory motion again during a trial. Trials were omitted and later collected again when illusory motion perception did not stop at the first-image offset. We collected data from 30 trials for each combination of two stimuli and 11 ISIs (i.e., 660 trials in total) for each observer.

In addition, we also measured the time courses of motion perceived in the Ouchi and Enigma illusions using the same method noted above. However, these illusions did not show clear decreasing trends in the occurrence rates during the first $10 \mathrm{~s}$ of stimulus presentation. Therefore, we did not test the effects of ISI and the second stimulus presentation with these illusions. We also excluded the judgment of the direction of illusory motion, because the Ouchi and Enigma illusion did not seem to have a tendency to move in a particular direction (clockwise or counterclockwise). Each illusion was tested in 28 trials. The order of testing of the two illusions was randomized.

\section{Results}

According to the participant's keypressing for each trial, we recorded the sequence of the beginning and end of illusory motion on a time scale, as shown in Fig. 4. For each of the FW and modified-FW stimuli, data sequences from 30 trials were acquired for the second image presentation under each ISI condition. For the first image presentation, data sequences from 330 trials (i.e., 30 trials $\times 11$ ISI conditions) were acquired under all 11 ISI conditions. Therefore, the accumulation of the time sequence triggered by the first image presentation produced the envelopes indicating the changes in the percentages of perceived illusory motion. For example, if $5 \mathrm{~s}$ after the first-image onset one perceived illusory motion in 33 out of the 330 trials, that particular point in time would have a $10 \%$ occurrence rate.

The results in Fig. 4 clearly show that the illusory motion started immediately after the first image presentation, at least under the FW stimulus conditions, but that the percentages of the perceived illusory motion start decreasing after a few seconds. This confirms that the illusory motion stopped within several seconds after the first image presentation. In some cases, rather long latencies were needed to perceive the illusory motion in full strength (participant N.A.). This result is consistent with interviews with the participant, who said that it took time to make direction judgments.

We calculated the durations of the perceived illusory motion during the first image presentation for each stimulus and for each participant. The average durations of the perceived illusory motion from the first FW stimulus presentation were $2.52,5.90,0.65,1.36,3.01$, and $3.51 \mathrm{~s}$ for participants F.S., N.A., N.M., O.R., S.A., and T.E., 
FW stimulus

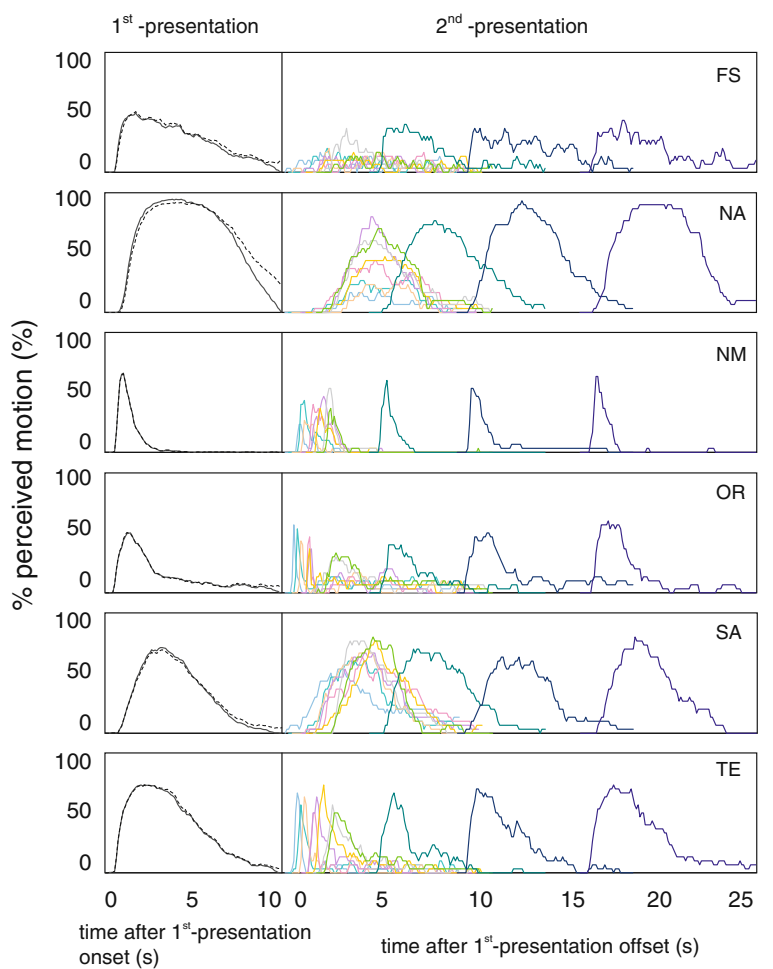

Fig. 4 Percentages of perceived motion as a function of time. The horizontal axis indicates the times after the onset or the offset of the first image presentation. The vertical axis indicates the percentage of perceived motion over 330 trials for the first presentation and 30 trials for the second presentation. The graph shows the time course of the motion perception decreasing with time and recovering with interstimulus interval (ISI). Motion perception recovers increasingly better

respectively. The average durations of the illusory motion from the first-modified-FW-stimulus presentation were $3.26,1.41,0.79,1.85,3.02$, and $3.08 \mathrm{~s}$ for participants $\mathrm{F}$. S., N.A., N.M., O.R., S.A., and T.E., respectively. The correlation coefficient between these durations was only .17. Although the individual differences in the illusory motion durations were rather large, the durations were less than $6 \mathrm{~s}$ for the first image presentation of either stimulus type. The points of peak probabilities in time were not different between the two illusions. Under the FW stimulus condition, the peaks occurred 1.7, 4.0, 1.0, 1.4, 3.2, and $2.2 \mathrm{~s}$ after the first-image onset for participants F.S., N.A., N.M., O.R., S.A., and T.E., respectively. On the other hand, for the modified-FW stimulus condition, the peaks were at $1.5,5.7,1.0,1.5,3.2$, and $2.5 \mathrm{~s}$ after the first stimulus onset for participants F.S., N.A., N.M., O.R., S.A., and T.E., respectively. The correlation coefficient between these peak positions was .97 . We also calculated correlation coefficients between the probability curves from the two illusions for each participant. We sampled illusion-occurrence probabilities at every 0.1 second, resulting in 100 samples in total for each curve. The correlation coefficients were
modified-FW stimulus

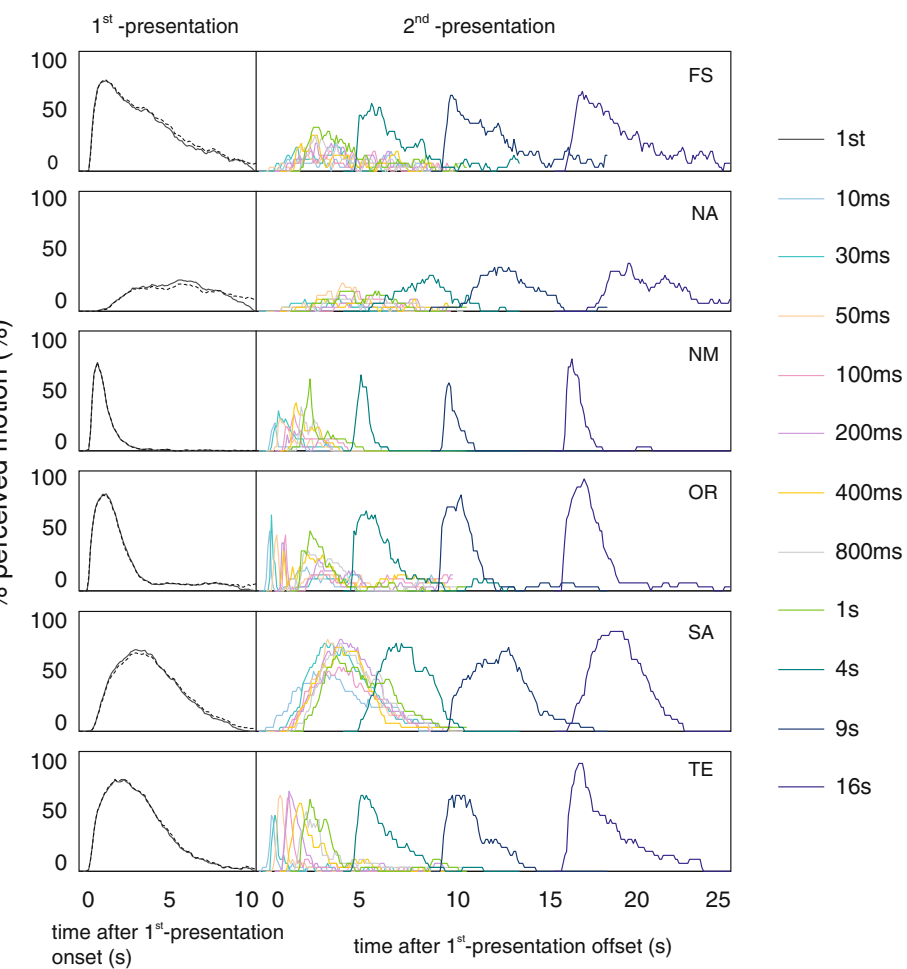

with longer ISI durations. Each color in the legend indicates one of the 11 ISIs. Dashed lines indicate the percentages of perceived motion for 330 trials, including the trials on which the illusory motion did not halt at the first presentation offset. Even when the no-halt trials are included, the trends of the response curves for the first presentation are nearly the same

$.97, .82,1.00, .98,1.00$, and .99 for participants F.S., N.A., N.M., O.R., S.A., and T.E. This demonstrates that the two response probability curves were nearly the same, except for those acquired from participant N.A. Because the nearzero responses after several seconds might have made the correlation very high, the correlation coefficients were recalculated for data only within $5 \mathrm{~s}$ after the first stimulus onsets. The recalculated correlation coefficients were $.97, .90,1.00, .98$, 1.00, and .99 for participants F.S., N.A., N.M., O.R., S.A., and T.E. This again shows that the two response probability curves are very similar in their quality. These analyses demonstrate that the measured strengths of the two illusions vary with participants, but that the changes in illusory persistence over time are quite similar between the two illusions.

The results also clearly show that the perceived illusory motion from the second image presentation recovered when an ISI was inserted. Even when the ISI was quite short-for instance, less than $1 \mathrm{~s}$ - the illusory motion recovered from the halt. When the ISI was longer than $1 \mathrm{~s}$, the recovery of the illusory motion perception became greater with increasing ISI.

Figure 5 shows the proportions of illusory motion perceived in the second image presentation, as compared 


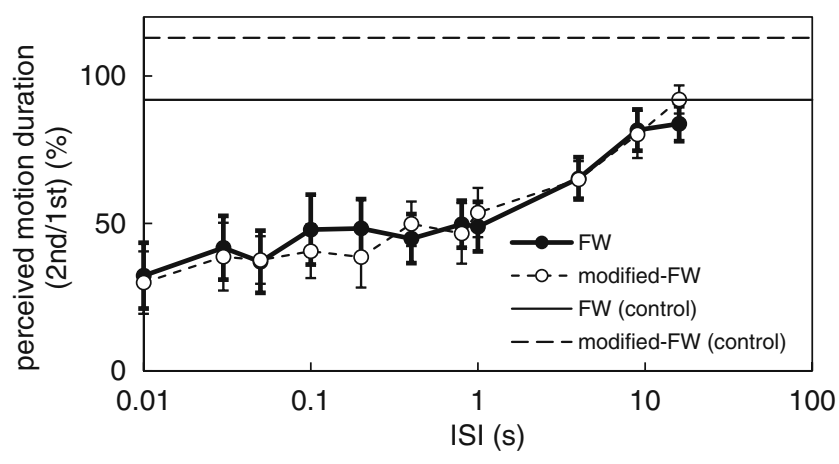

Fig. 5 Durations of perceived motion as a function of ISI. The horizontal axis shows the ISI in seconds on a log scale. The vertical axis shows the perceived motion duration following the second image presentation divided by that following the first image presentation. The straight lines at the top of the figure represent illusion strength during control trials. The error bars indicate standard errors

to that in the first image presentation, for each ISI. While the main effect of ISI was significant $[F(10,50)=20.13, p<.01]$, no significant difference was found between the stimulus types $[F(1,5)=0.14, p>.05]$. The interaction between the two factors was also not significant $[F(10,50)=0.70$, $p>.05]$. These statistics demonstrate that the recovery of the illusory motion was affected by the length of the ISI and that the time course of the recovery phase was not different between the two types of stimuli.

By contrast, the durations of perceived motion in the control conditions were almost the same between the first and second image presentations, as shown in Fig. 6. When the second image was presented at a different retinal location than the first presentation, the perceived motion duration recovered almost $100 \%$ (Fig. 5, horizontal lines). Even though the stimulus was presented without an ISI, the recovery was at the same level as (or higher than) in the 16-s ISI condition. Presenting the second image at a different retinal position thus revitalized illusory motion that had come to a halt during the first image presentation at the original retinal position.

Figure 7 shows the perceived illusory motion in the Ouchi and Enigma illusions. The curves in the figure were plotted in the same way as for the FW and modified-FW stimuli, although the ISI and the second-image-presentation periods were not included. In the Ouchi illusion, the occurrence probabilities were rather low. One reason for this could be that the size of the squares in the Ouchi figure was nearly the same as the size of the small elements in the FW or the modified-FW stimulus. As a result, the spatial frequency was low, as compared to the optimal frequency reported by Ashida (2002). It is more conceivable, though, that the infrequent occurrence of the illusion resulted from the existence of the fixation point, which restricted eye movements. As a result, microsaccades or fixation instability did not cause the illusion effectively, as evidenced by its random occurrence and inconsistent trend curves among the participants. As for the Enigma conditions, rather than showing a decrease after a few seconds, the probability of perceiving illusory motion increased. The probabilities did not decrease until the end of the stimulus presentation. In summary, the
Fig. 6 Percentages of perceived motion in the control conditions
FW stimulus

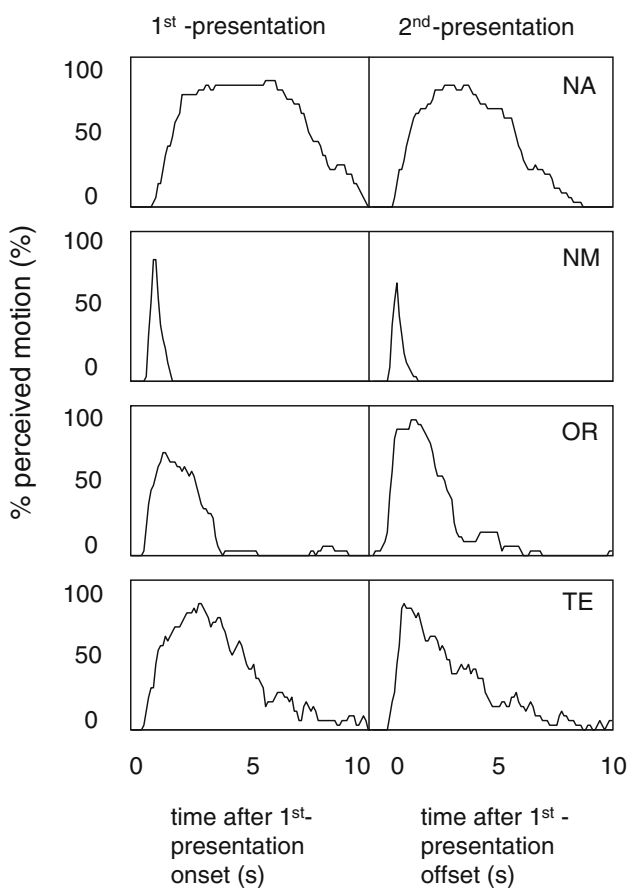

modified-FW stimulus

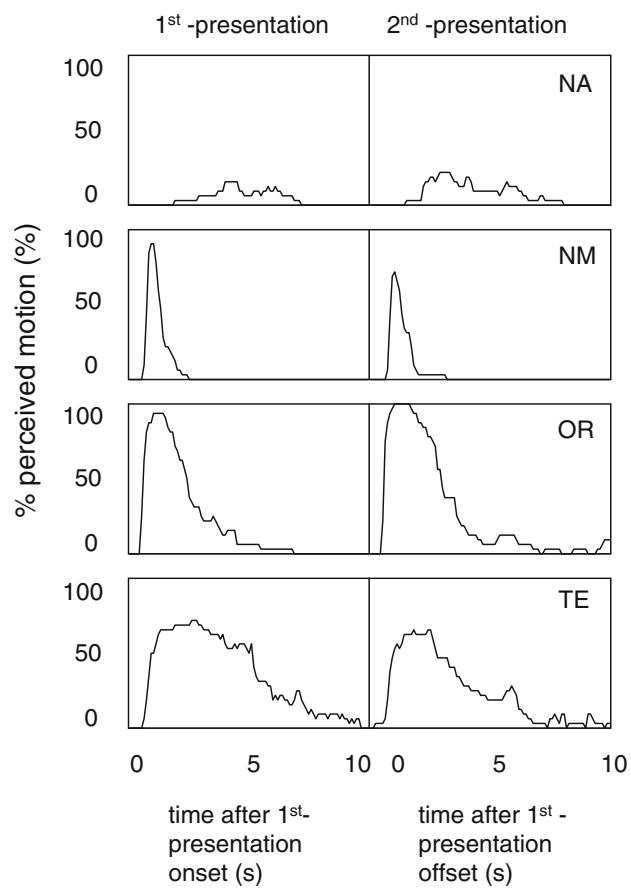


Fig. 7 Percentages of perceived motion as a function of time for the Ouchi and Enigma illusions. The horizontal axis indicates the time after the onset of the stimulus presentation. The vertical axis indicates the percentage of perceived motion from 28 trials
Ouchi

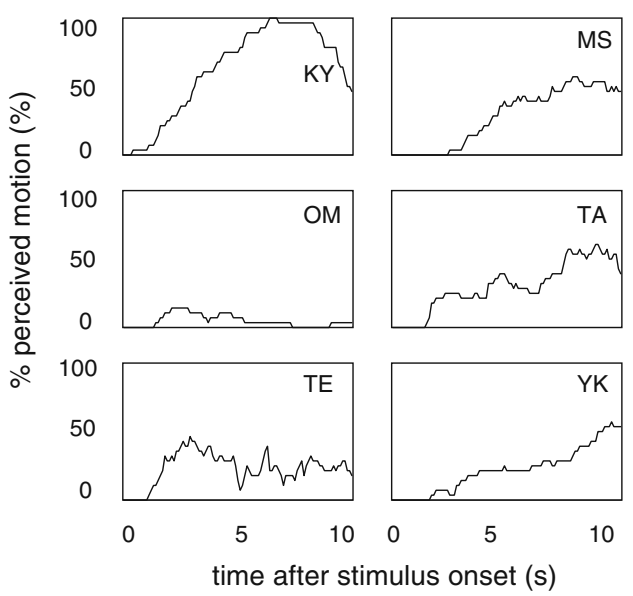

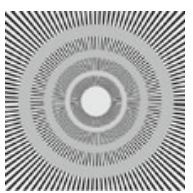

Enigma

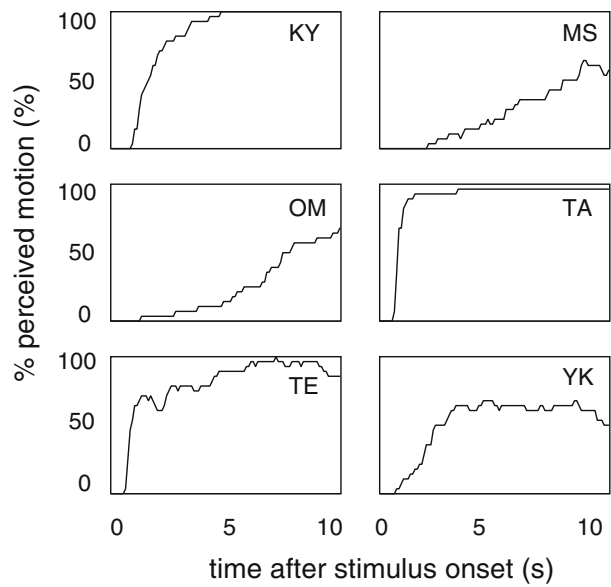

trend curves acquired from the Ouchi and Enigma illusion conditions showed a clear contrast with those obtained from the FW and modified-FW stimulus conditions.

Figure 8 shows a comparison of the temporal properties of the four stimuli. It shows normalized percentages of perceived motion as a function of time after the stimulus onset. These percentages were obtained by normalizing the percentages of perceived motion (in Figs. 4, 6, and 7); that is, we regarded the value at the peak of the time course as $100 \%$ for each observer and then averaged the normalized percentages over the 6 participants. The percentages from

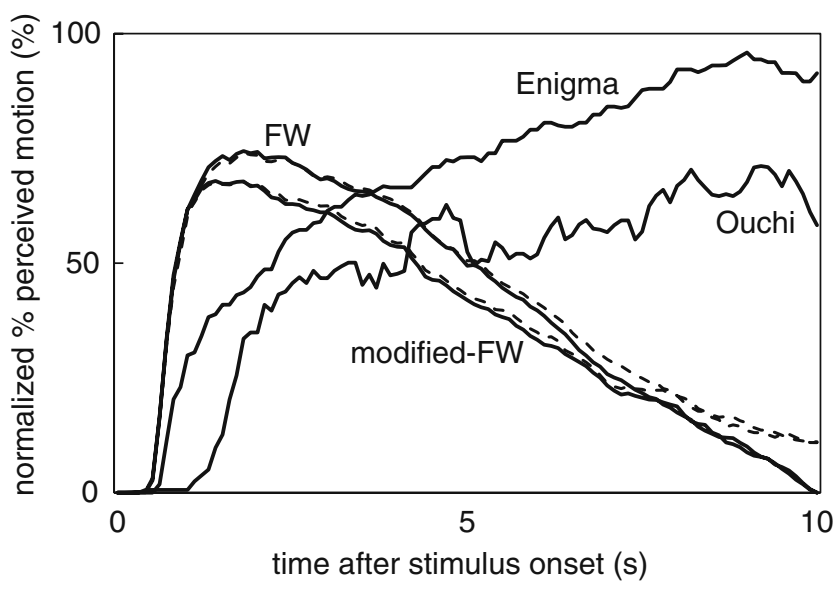

Fig. 8 Time courses for the four stimuli: FW (first presentation), modified FW (first presentation), Ouchi, and Enigma. The horizontal axis indicates the time after stimulus onset. The vertical axis indicates the average of the normalized percentages of perceived motion over participants. Dashed lines indicate the time courses for 330 trials, including the trials on which the illusory motion did not halt at offset of the first presentation, for the FW or the modified-FW stimulus the FW and modified-FW stimulus conditions decreased with time. Their temporal properties are rather similar, and quite different from the curves obtained with the Ouchi and Enigma stimuli. This strongly indicates that the changes in illusion strength for all four stimuli cannot be accounted for by a single source, such as fixation instability.

\section{Discussion}

\section{General remarks}

In this study, we demonstrated the whole time course of the illusory motion perceived in static images introduced by Fraser and Wilcox (1979) and Kitaoka and Ashida (2003), including a recovery phase after the illusory motion stopped. Our experiment shows that the illusory motion stopped in a few seconds, while the complete recovery of the illusory motion perception took over $16 \mathrm{~s}$ after the stimulus was erased. Motion perception gradually recovered with increasing ISI when the stimulus was presented again (second image presentation) in the same retinal location. In contrast, the immediate second image presentation at a different retinal location showed perfect recovery of the illusory effect. We suggest that neural adaptation affects both the FW illusion and the modified FW illusion. Despite a recent study (Cantor, Tahir, \& Schor, 2010) in which the researchers argued that an optical mechanism causes the modified FW illusion, in view of the time courses of the illusory motion, an explanation in terms of neural adaptation seems plausible at this stage of research. The time courses shown in Fig. 4 are easily interpreted in 
terms of the adaptation hypothesis (Backus \& Oruç, 2005) and provide quantitative validation of earlier qualitative observations. We suggest that the perceptual change in the illusion strength corresponds to each of the phases in local luminance and contrast adaptation: an adaptation phase (the period of seeing illusory motion), a saturated phase (the period of the halt of the illusory motion), and a recoveryfrom-saturation phase (the ISI).

We informally observed that when presenting the second image at a different retinal position without erasing, the first image looked duller and lower in contrast than the second image, which instead looked clear and high in contrast. This difference in perceived vividness may suggest that local adaptation to contrast and/or luminance actually took place. In addition, the illusory motion in this case was perceived more strongly for the second image presentation. Although the first image sometimes seemed to move when the second image was presented, the subjective strength of the illusion was much weaker. These observations may support the above-noted conclusion that a local adaptation process determined the illusion strength.

\section{Effect of small eye movements on local adaptation}

Microsaccades and drift eye movements may play an important role in avoiding adaptation and delaying saturation. However, Murakami et al. (2006) denied this possibility, because they did not find a correlation between the illusion strength and the frequency of microsaccades. One possible explanation for their result is suggested by the response curves shown in Figs. 4 and 6. Their cancellation experiment used a stimulus duration of $0.5 \mathrm{~s}$. It is evident that the illusion is strongest at this moment, if participants' reaction times are taken into account. Thus, there is no need to refresh the retinal image to maintain the illusory motion by microsaccades within $0.5 \mathrm{~s}$. Murakami et al.'s subsidiary experiment used a 15-s stimulus duration and acquired very close ratings of the illusion strength from both free viewing and "steady fixation" conditions. The researchers noted that steady fixation did not eliminate the illusion. However, their fixation spot was about $1^{\circ}$ in diameter. Therefore, it is possible that small eye movements within $1^{\circ}$ had a large effect on maintaining the illusory motion perception and avoiding its halt in their study. In contrast, the present study, in which the participants fixated on a cross, shows that the illusion stopped within a few seconds (Figs. 4 and 6). Thus, the correlation between illusion strength and the fixation instability (mainly drifts) found by Murakami et al. might indicate that great fixation instability tends to slow the local adaptation to contrast and luminance. It is also possible that the large individual differences in perceived motion duration obtained in the present study were caused by individual differences in fixation instability that assisted some participants in avoiding local adaptation.

\section{Ouchi and Enigma illusions}

We also measured the time courses of perceived motion in the Ouchi and Enigma illusions. Motion in both of these illusions has been suggested to involve eye movements. For example, the Ouchi illusion is considered to be caused by retinal slips arising from eye movements or from motion of the figure itself. The Enigma illusion is influenced by microsaccades (Troncoso et al., 2008). As is shown in Fig. 7, the curves representing percentages of perceived illusory motion as a function of time from stimulus onset are quite different from those of the FW and modified-FW stimuli. It is obvious that the Ouchi and Enigma illusions show no decrease in their perceived motion probabilities during the first $10 \mathrm{~s}$ after stimulus onset. In addition, the Ouchi illusion seems to arise at rather random timings. We guess that the illusion is accidentally caused by relatively large drift eye movements. On the other hand, the Enigma illusion seems to gradually grow in strength or can be observed from the stimulus onset. Although this illusion is related to microsaccades (Troncoso et al., 2008), it is perceived not only during the moments of microsaccades but also throughout a subsequent time period.

In both the Ouchi and Enigma illusions, luminance gratings are also necessary to produce the illusory motion. However, the illusions do not seem to suffer from local luminance and/or contrast adaptation. It is also possible that they could recover from saturation quickly through very small eye movements that would not be sufficient to revive motion with the FW and modified-FW stimuli. In view of these results, we guess that the illusions produced by Fraser and Wilcox (1979) and Kitaoka and Ashida (2003) are more directly affected by the progress of adaptation to luminance gratings. Billino et al. (2009) showed that the "Rotating Snakes" illusion is strongly affected by aging and suggested a contribution of some specific physiological process. They demonstrated that there is no correlation between the "Rotating Snakes" and Enigma illusions in their probabilities of occurrence. Tomimatsu, Ito, Seno, and Sunaga (2010) also reported a difference among the illusions we have studied. Tomimatsu et al. added smooth motion to the illusion stimuli and found that the "Rotating Snakes" illusion was dramatically decreased by the smooth motion. By contrast, the streaming in the Enigma illusion did not apparently decrease, and the Ouchi illusion was even enhanced by adding smooth motion. The present results, showing differences in the time courses of perceived motion in the illusions, are consistent with the previous results (Billino et al., 2009; Tomimatsu et al., 2010).

Difference between Fraser and Wilcox (1979) and Kitaoka and Ashida (2003)

The results from the present study strongly suggest that the FW and modified FW illusions tested by our stimuli were 
affected by the same adaptation mechanism. First, the probability curves for the first stimulus presentation were quite similar between the two illusions. Second, there were no differences in the recovery phase between the two. However, we do not insist that the two illusions are completely the same in their motion induction mechanisms.

In addition, we found that the motion direction perceived in the FW stimulus sometimes was not constant, as Fraser and Wilcox (1979) had also noted, even within a participant. It is possible that the dark-gray and light-gray areas in the FW stimulus assimilated into the mid-gray background. As a result of this process, the stimulus might have looked like a simple configuration of black and white, which produced a bistable direction of illusory motion. When the background was mid-gray, a perceived motion direction from light to dark was dominant. This is the direction opposite the one reported by Faubert and Herbert (1999) and Naor-Raz and Sekuler (2000). Actually, 51\%$99 \%$ (mean $=72 \%$ ) of the total illusory motion duration in our FW stimulus was perceived in the light-to-dark direction, depending on the participant. The stimulus was therefore not strictly bistable. At least, we can say that motion in the FW illusion can occur in the direction from light to dark, and the dominant direction might be determined by the stimulus contrast with the background. Here we used a mid-gray background, whereas previous studies (Faubert \& Herbert, 1999; Naor-Raz \& Sekuler, 2000) seem to have used a white background.

On the other hand, for the modified-FW stimulus, the perceived motion direction was almost constant (from black to dark gray, white, and light gray). In total, 65\%-92\% (mean = $84 \%$ ) of the illusory motion duration was perceived in this direction, again depending on the participant. The two types of stimuli may have commonalities, at least in the effect of contrast and/or luminance adaptations, yet they may differ in how their perceived motion direction is determined.

Backus and Oruç (2005) suggested that the modified FW illusion is produced by quick contrast adaptation and that the FW illusion is produced by slow luminance adaptation. However, we did not find a difference in the peak points of perceived illusory motion in time. One cause for this might be the reaction time of the participants. That is, the delay of the key responses might have hidden a possible difference. However, even for the participants N.A. and S.A., whose peak points in time were more than $2 \mathrm{~s}$ from stimulus onset, the peak point of the modified-FW stimulus was not earlier than that of the FW stimulus. With the measuring methods used here, we thus could not demonstrate that the modified FW illusion was caused by a quicker process than the original $\mathrm{FW}$ version.

Another possible argument for not finding a difference in the peak points of the two illusions is that our FW stimulus was not processed like a typical FW illusion. Our FW stimulus consisted of four steps of luminance profiles corresponding to those of the modified-FW stimulus. This simplification might have weakened the characteristics of the FW illusion, causing it to be treated as a version of the modified-FW stimulus. However, we acquired the same characteristic on the perceived motion directions from the FW stimulus as reported by Fraser and Wilcox (1979) and found a participant who perceived weak motion solely for the modified-FW stimulus. Thus, we believe that our stimuli had retained the essential components of the illusions.

On the cause of the illusions

We suggest that the change in perceived motion in both illusions represents the different phases in a local luminance and contrast adaptation process: adaptation (the first image presentation), saturation (or complete adaptation; the halt of the illusory motion), and recovery from saturation (the ISI period). This theory could be in line with the hypothesis of Backus and Oruç (2005) that the process of the local luminance and contrast adaptation is the direct cause of the illusory motion. According to their hypothesis, the halt of the illusory motion should be interpreted as indicating a state of complete adaptation. Eye movements could refresh the state of the local adaptation, resulting in delay of the halt or recovery of the illusory motion.

However, we should be cautious about rejecting the eye movement hypothesis (Murakami et al., 2006), because it is possible to argue that adaptation to local luminance and contrast reduces the effect of the fixation instability that produces local motion signals. Naor-Raz and Sekuler (2000) reported that the magnitude estimation of the strength in the FW illusion depended on the stimulus contrast. If this is the case, even though the progress of the local adaptation process does not directly cause the illusory motion, the effective contrast due to contrast adaptation would modulate or attenuate the strength of the effect of eye movement causing the local motion signals. According to this hypothesis, the halt of the illusory motion would be interpreted as a state of reduced effective contrast (or luminance), resulting in minimizing local motion detection.

At present, it is difficult to differentiate the two hypotheses - that is, that adaptation either causes or attenuates the illusions-because the time courses of the phenomenon should be similar between the two hypotheses. However, it is evident that the illusion strength curves obtained here represent the same adaptation process in both the FW and modified FW illusions, and that the strength of the perceptual illusion in both stimuli is determined by the state of local adaptation. 
Author Note This research was partly supported by KAKENHI (Grants 08 J03360, 191030032, 19653083, and 2653092) and by a Grant-in-Aid for the 21st Century COE Program.

\section{References}

Anstis, S. M. (1970). Phi movement as a subtraction process. Vision Research, 10, 1411-1430.

Ashida, H. (2002). Spatial frequency tuning of the Ouchi illusion and its dependence on stimulus size. Vision Research, 42, 1413-1420.

Backus, B. T., \& Oruç, I. (2005). Illusory motion from change over time in the response to contrast and luminance. Journal of Vision, $5,1055-1069$.

Beer, A. L., Heckel, A. H., \& Greenlee, M. W. (2008). A motion illusion reveals mechanisms of perceptual stabilization. PLoS ONE, 3, e2741.

Billino, J., Hamburger, K., \& Gegenfurtner, K. R. (2009). Age effects on the perception of motion illusions. Perception, 38, 508-521.

Cantor, C. R. L., Tahir, H. J., \& Schor, C. M. (2010). Is the rotating snakes an optical illusion? (VSS Abstract). Journal of Vision, 10 (7), $824 \mathrm{a}$

Conway, R. B., Kitaoka, A., Yazdanbakhsh, A., Pack, C. C., \& Livingstone, M. S. (2005). Neural basis for a powerful static motion illusion. Journal of Neuroscience, 25, 5651-5656.

Faubert, J., \& Herbert, A. M. (1999). The peripheral drift illusion: A motion illusion in the visual periphery. Perception, 28, 617-621.

Fermüller, C., Pless, R., \& Aloimonos, Y. (2000). The Ouchi illusion as an artifact of biased flow estimation. Vision Research, 40, 77-95.

Fraser, A., \& Wilcox, K. J. (1979). Perception of illusory movement. Nature, 281, 565-566.
Hamburger, K. (2007). Apparent rotation and jazzing in Leviant's enigma illusion. Perception, 36, 797-807.

Hisakata, R., \& Murakami, I. (2008). The effects of eccentricity and retinal illuminance on the illusory motion seen in a stationary luminance gradient. Vision Research, 48, 19401948.

Kitaoka, A. (2003). "Rotating snakes" (Bitmap figure). Retrieved 5 February, 2010, from www.ritsumei.ac.jp/ akitaoka/index-e.html

Kitaoka, A., \& Ashida, H. (2003). Phenomenal characteristics of the peripheral drift illusion. Vision, 15, 261-262.

Kitaoka, A., \& Ashida, H. (2007). A variant of the anomalous motion illusion based upon contrast and visual latency. Perception, 36, $1019-1035$.

Leviant, I. (1996). Does "brain-power" make Enigma spin? Proceedings of the Royal Society B, 263, 997-1001.

Murakami, I., Kitaoka, A., \& Ashida, H. (2006). A positive correlation between fixation instability and the strength of illusory motion in a static display. Vision Research, 46, 24212431.

Naor-Raz, G., \& Sekuler, R. (2000). Perceptual dimorphism in visual motion from stationary patterns. Perception, 29, 325-335.

Spillmann, L., Heitger, F., \& Schuller, S. (1986). Apparent displacement and phase unlocking in checkerboard patterns. Paper presented at the 9th European Conference on Visual Perception, Bad Nauheim, Germany.

Tomimatsu, E., Ito, H., Seno, T., \& Sunaga, S. (2010). The Rotating Snakes in smooth motion do not look as rotating. Perception, 39, 721-724.

Troncoso, X. G., Macknik, S. L., Otero-Millan, J., \& Martinez-Conde, S. (2008). Microsaccades drive illusory motion in the Enigma illusion. Proceedings of the National Academy of Sciences, 105, 16033-16038. 